\title{
Maximal domains for strategy-proof or Maskin monotonic choice rules
}

Citation for published version (APA):

Bochet, O. L. A., \& Storcken, A. J. A. (2006). Maximal domains for strategy-proof or Maskin monotonic choice rules. METEOR, Maastricht University School of Business and Economics. METEOR Research Memorandum No. 003 https://doi.org/10.26481/umamet.2006003

Document status and date:

Published: 01/01/2006

DOI:

10.26481/umamet.2006003

Document Version:

Publisher's PDF, also known as Version of record

\section{Please check the document version of this publication:}

- A submitted manuscript is the version of the article upon submission and before peer-review. There can be important differences between the submitted version and the official published version of record.

People interested in the research are advised to contact the author for the final version of the publication, or visit the DOI to the publisher's website.

- The final author version and the galley proof are versions of the publication after peer review.

- The final published version features the final layout of the paper including the volume, issue and page numbers.

Link to publication

\footnotetext{
General rights rights.

- You may freely distribute the URL identifying the publication in the public portal. please follow below link for the End User Agreement:

www.umlib.nl/taverne-license

Take down policy

If you believe that this document breaches copyright please contact us at:

repository@maastrichtuniversity.nl

providing details and we will investigate your claim.
}

Copyright and moral rights for the publications made accessible in the public portal are retained by the authors and/or other copyright owners and it is a condition of accessing publications that users recognise and abide by the legal requirements associated with these

- Users may download and print one copy of any publication from the public portal for the purpose of private study or research.

- You may not further distribute the material or use it for any profit-making activity or commercial gain

If the publication is distributed under the terms of Article $25 \mathrm{fa}$ of the Dutch Copyright Act, indicated by the "Taverne" license above, 


\title{
Maximal Domains for Strategy-Proof or Maskin Monotonic Choice Rules* Olivier Bochet ${ }^{\dagger}$ and Ton Storcken ${ }^{\ddagger}$
}

\author{
December 2005
}

\begin{abstract}
Domains of individual preferences for which the well-known impossibility theorems of Gibbard-Satterthwaite and Muller-Satterthwaite do not hold are studied. To comprehend the limitations these results imply for social choice rules, we search for the largest domains that are possible. Here, we restrict the domain of individual preferences of precisely one individual. It turns out that, for such domains, the conditions of inseparable pair and of inseparable set yield the only maximal domains on which there exist non-dictatorial, Pareto-efficient and strategy-proof social choice rules. Next, we characterize the maximal domains which allow for Maskin monotone, non-dictatorial and Pareto-efficient social choice rules.
\end{abstract}

\section{Introduction}

The two most negative results on the decentralization of social choice functions are, respectively, the Gibbard-Satterthwaite (Gibbard (1973), and Satterthwaite 1975) and the Muller-Satterthwaite (Muller and Satterthwaite,

${ }^{*}$ Corresponding author: o.bochet@algec.unimaas.nl. Mailing address: Maastricht University, department of economics, P.O. Box 616. 6200 MD Maastricht, The Netherlands.

${ }^{\dagger}$ Maastricht University and CORE.

${ }^{\ddagger}$ Maastricht University. 
1977) theorems. The Gibbard-Satterthwaite theorem states that over an unrestricted domain of linear orderings-and with at least three alternativesany surjective and strategy-proof social choice function is dictatorial. On the other hand, the Muller-Satterthwaite theorem, by establishing the connections between strategy-proofness and Maskin monotonicity, states that any unanimous and Maskin monotonic social choice function is dictatorial.

These two theorems have a damaging impact on decentralization. Strategyproofness and Maskin monotonicity are, respectively, necessary conditions for dominant strategy implementation and Nash implementation. If a planner restricts himself to institutions corresponding to normal game forms having a unique equilibrium at every preference profile, then only trivial social choice functions are decentralizable.

However, the two results strongly rely on the assumption of unrestricted domain of preferences. Restricted domains have delivered possibility results on strategy-proof and Maskin monotonic social choice functions. ${ }^{1}$ For instance, if preferences are quasi-linear with respect to a numeraire good, then Clark-Groves mechanisms are strategy-proof. ${ }^{2}$ If preferences are singlepeaked, then generalized median voting rules are strategy-proof (see e.g. Moulin, 1980). Finally, in exchange or production economies, if the domain of preferences is such that there always exist a unique Walrasian equilibrium in the interior of the feasible set, then the Walrasian social choice function is Maskin monotonic and obviously non-dictatorial. ${ }^{3}$

In this paper, we work with the abstract social choice model. We are interested in the maximal preference domains under which there exists social choice functions that escape the Gibbard-Satterthwaite and the MullerSatterthwaite theorems. This question is not new, at least for the strategyproof case. In the literature, the approach that is usually followed is to take a possibility domain and to find the maximal enlargement of this domain so that the possibility result still holds. For instance, this is the case of Barberà, Sonnenschein and Zhou (1994); Barberà, Gul and Stacchetti (1994); Serizawa (1995); Serizawa and Ching (1998); or Masso and Neme (2001). Each paper deals with entire restricted domain of preferences. Moreover, as

\footnotetext{
${ }^{1}$ There is a vast literature providing possibility results in various models. We voluntarily only make reference to a few papers.

${ }^{2}$ Though, as shown in Green and Laffont (1979), their lack of budget-balancedness cannot be overcome. As a consequence, Clark-Groves mechanisms are not fully efficient.

${ }^{3}$ Obviously, in such a case, the Walrasian social choice function is not strategy-proof (see e.g. Hurwicz, 1972).
} 
far as we are aware of, there are no papers that deals with maximal domains for non-trivial Maskin monotonic social choice functions. Closely related but different questions were posed by Kalai and Muller (1978) and Kalai and Ritz (1980). They studied the general conditions for domains which admit the existence of non-dictatorial Arrow-type social welfare functions.

Our approach is different. Instead of allowing that any agent's preference domain be restricted, we restrict the preference domain of precisely one agent. By doing so, we look for necessary and sufficient conditions that guarantee a possibility result. We identify two conditions that are both necessary and sufficient for the existence of non-trivial social choice functions. The social choice function $K$ we identify is a hierarchical rule. In that sense, the rule is almost dictatorial. The conditions we identify are respectively strategy-proof admissibility and Maskin admissibility.

We then examine the maximality of this domain restrictions. We study, in turn, strategy-proofness and Maskin monotonicity since the conditions we obtain are different. For strategy-proofness, we find that a domain of preferences is a maximal possibility domain if and only the restricted set of preferences of agent 1 has one inseparable pair or one inseparable set. The notion of inseparable pair is well-known in relation with non-dictatorial Arrow-type welfare functions (see e.g. Kalai and Ritz, 1980). Just to fix idea, let us briefly discuss the notion of inseparable pair and inseparable set. We say that an agent has an inseparable pair if there exist two alternatives $x$ and $y$ such that whenever $x$ is ranked best, then $y$ is second-best. ${ }^{4}$ Suppose for instance that a board of managers has two vacancies. Current members of the board are contemplating several candidates. Among those are $b$, a bossy individual, and $w$ a wimp that is afraid of $b$. Then, an agent $i$ could rank $b$ at the top and $w$ second because he expects $w$ to be obedient and to copy $b^{\prime} s$ decisions-the power of $b$ would then be increased. ${ }^{5}$ Clearly, agent $i$ has an inseparable pair $(b, w)$.

An agent has an inseparable set if there exist a set of alternatives $B$ of cardinality at least equal to three-such that all preferences with a best alternative in $B$ ranks the alternatives in $B$ adjacent to each other (while

\footnotetext{
${ }^{4}$ The definition of inseparable pair used by Kalai and Ritz (1980) is stronger: it says that for all preferences where $x$ is preferred to $y$, these two are ranked adjacent to each other.

${ }^{5}$ On the other hand, for different preferences, this agent $i$ may like a strong candidate but dislike $b^{\prime} s$ bossiness and $w^{\prime} s$ obedience. Then, $b$ and $w$ are not necessarily ranked adjacent to each other because $i$ does not like when the power of $b$ is increased.
} 
the ranking of alternatives both within $B$ and the complement of $B$ can be reversed from one profile to another) ${ }^{6}$ For instance, suppose that there are two parties, left and right, involved in an election where several positions have to be distributed (e.g. prime minister versus lower ranked ministers). ${ }^{7}$ Agents have to vote for one of the two parties and have to rank candidates within each party according to the position they would like them to have. There, it is natural to assume that agents rank individuals from the same party adjacent to each other. Then, every agent has an inseparable set. ${ }^{8}$ On the other hand, in order to escape the Gibbard-Satterthwaite theorem, we only need one agent to satisfy the domain restriction imposed by inseparable pair or inseparable set.

The notion of inseparable set goes at least back to Storcken (1989). It follows that, in case of precisely three alternatives, if one arbitrary chosen preference is no longer admissible for one arbitrary chosen agent-yielding an inseparable pair for this agent-then on this domain neither the negative result of Gibbard Satterthwaite nor that of Muller Satterthwaite can be deduced.

For Maskin monotonicity, the condition is more intricate and relies on the existence of disjoint subsets of the sets of alternative and the existence of an asymmetric and transitive relation on the set of alternatives. However, like in the case of the maximal strategy-proof possibility domains, the result spells out a characterization of maximal Maskin monotone possibility domains in terms of a set of pairs of alternatives on which the coalition of agents whom sets of preferences are not restricted are decisive

The plan of the paper is as follows. In section 2, we introduce the model and the necessary definitions useful for the paper. Section 3 presents sufficient conditions for the existence of possibility results, as well as some connections between strategy-proofness and Maskin monotonicity. Then, section 4 establishes that these sufficient conditions are also necessary. Section 5 characterizes the maximal domain for strategy-proof, efficient and non-dictatorial social choice functions. Next, section 6 characterizes the maximal domain for Maskin monotonic, efficient and non-dictatorial social choice functions. Finally, section 7 provides some concluding remarks.

\footnotetext{
${ }^{6}$ Observe that if the cardinality of $B$ is equal to 2 , then we have in fact two inseparable pairs.

${ }^{7}$ Thus, each party has several candidates.

${ }^{8}$ Thus, the strategy-proof and Pareto-efficient rules that one may identify there will be less hierarchical than the one we identify.
} 


\section{The model}

There is a set of alternatives $A=\{1, \ldots, m\}$, with $m \geqslant 3$ and a set of agents $N=\{1, \ldots, n\}$ with $n \geqslant 2$.

Agents are endowed with a preference relation $R$ over elements of $A$ that is (strongly) complete, anti-symmetric and transitive; that is a preference relation is a linear order over alternatives. Let $L(A)$ denote the set of all these preferences and $L^{N}(A)$ be the set of possible preference profiles. For each $R \in L(A)$ and each $(x, y) \in A \times(A \backslash\{x\}), x \ldots=R$ means that $x$ is the best alternative at $R, \ldots x \ldots y \ldots=R$ means that $x$ is strictly preferred to $y$ at $R, \ldots x y \ldots=R$ means that $x$ is strictly preferred to $y$ at $R$ and there is no alternative in between; and finally $x \ldots y=R$ means that $x$ is the best alternative and $y$ is the worst alternative at $R$. Let $L_{x}(A)$ denote the set of linear orderings $R$ such that $x \ldots=R$.

For each relation $R$ on $A$, for each $x, y \in A,(x, y) \in R$ means that ....x...y... $=R$ or $x=y$, i.e. $R \subseteq A \times A$. The upper contour of an alternative $x$ at $R$ is defined as $u p(x, R)=\{y \in A:(y, x) \in R\}$ and the lower contour of $x$ at $R$ is defined as $\operatorname{low}(x, R)=\{y \in A:(x, y) \in R\}$. The complement of the upper contour of $x$ at $R$ is non-up $(x, R)=A-u p(x, R)$. Furthermore, let $I_{A}=\{(x, x): x \in A\}$ denote the identity relation on $A$.

To model restrictions of domains of individual preferences let $\emptyset \neq L^{i} \subseteq$ $L(A)$ be the domain of individual preferences of agent $i \in N$. From now on, we assume that $L^{i}=L(A)$ if $i \geqslant 2$ and $L^{1} \varsubsetneqq L(A)$. For each alternative $a \in A$ and set of preferences $V \subseteq L(A)$, let $L_{a}^{i}=L_{a}(A) \cap L^{i}$ and $V_{a}=V \cap L_{a}(A)$. For a coalition $M$, i.e. a subset of $N$, and alternatives $x, y$ in $A$, let $L_{x}^{M} \times$ $L_{y}^{N-M}$ denote the set of profiles $p$ such that $x \ldots=p(i)$ for all $i \in M$ and $y \ldots=p(i)$ for all $i \in N-M .^{9}$ We introduce the following restriction on domain of preferences.

Convexity: The domain $L^{i} \subseteq L(A)$ is convex if for all $R^{1}, R^{2}$ in $L^{i}$ and $R^{3}$ in $L(A)$

$$
R^{1} \cap R^{2} \subseteq R^{3} \subseteq R^{1} \cup R^{2} \text { implies that } R^{3} \in L^{i} .
$$

A social choice rule $K$ is a function from $L^{N}$ to $A$. The set of profiles $L^{N}$ is convex if for each agent $i \in N$, the preference domain $L^{i}$ is convex. For coalitions $M$ and profiles $p, q \in L^{N}$, the preference profile $p$ is said to be a $M$-deviation of a profile $q$ if $\left.p\right|_{N-M}=\left.q\right|_{N-M}$.

\footnotetext{
${ }^{9}$ We set $L_{x}^{\emptyset} \times L_{y}^{N}=L_{y}^{N}$ and $L_{x}^{N} \times L_{y}^{\emptyset}=L_{x}^{N}$.
} 
Inseparable pair: The set of preferences $L^{i}$ has an inseparable pair $(x, y) \in A \times(A \backslash\{x\})$ if $x y \ldots=R$ for each $R \in L_{x}^{i}$.

Inseparable set: Let $B$ be a subset of $A$, with $3 \leqslant \# B<m$. The set of preferences $L^{i}$ has an inseparable set $B$, with $3 \leqslant \# B<m$, if for each $b \in B$, for each $R \in L_{b}^{i}$, for each $a \in B$ and for each $c \in A-B$, we have $\ldots a \ldots c \ldots=R$.

So, for all preferences $R$ in $L^{i}$, if the best alternative at $R$ is in $B$, then $B$ is preferred to $(A-B)$ at $R$. Note that this condition trivially holds for the empty set, any singleton set and the set $A$ itself. Therefore these are excluded. Furthermore, if $B$ consists of precisely two alternatives, then having an inseparable set means having two inseparable pairs, which explains why sets with cardinality 2 are excluded in the definition of inseparable set.

The notion of inseparable pair is well-known see e.g. Kalai and Ritz (1980). Although there it is a slightly stronger condition. That is $L^{i}$ has an inseparable pair $(x, y)$ if for all $R \in L^{i}$ if $\ldots x \ldots y \ldots=R$, then $\ldots x y \ldots=R$. Here we only need this inseparability if $x$ is top alternative, because the almost dictatorial rule depends mainly on the top alternatives of agent 1 , the agent with the restricted preference set. A similar remark as for inseparable pair holds for inseparable set as defined in Storcken (1989).

The following conditions for choice rules are well-known. We just rephrase these using the notation at hand.

Non-dictatorship: The choice rule $K$ is non-dictatorial if for each agent $i \in N$, there is a profile $p \in L^{N}$ such that $K(p) \ldots \neq p(i)$.

Pareto efficiency: The choice rule $K$ is Pareto-efficient if for each $(x, y) \in A \times A$ and each $p \in L^{N}$ such that for all agents $i \in N, \ldots x \ldots y \ldots=p(i)$, then $K(p) \neq y$.

Strategy-proofness: The choice rule $K$ is strategy-proof if for each agent $i \in N$ and each $p, q \in L^{N}$ such that $q$ is an $\{i\}$-deviation of $p$, we have that either $K(p)=K(q)$ or $\ldots K(p) \ldots K(q) \ldots=p(i)$.

Intermediate strategy-proofness: The choice rule $K$ is intermediate strategy-proof if for each coalition $M \subseteq N$ and for each profile $p \in L^{N}$, such that there is a preference $R \in L(A)$ with $p(i)=R$ for all $i \in M$, and all $M$-deviations $q \in L^{N}$, it holds that $\ldots K(p) \ldots K(q) \ldots=R$ or $K(p)=K(q)$. 
Maskin monotonicity: The choice rule $K$ is Maskin monotonic if for each $p, q \in L^{N}$,

$\operatorname{low}(K(p), p(i)) \subseteq \operatorname{low}(K(p), q(i))$ for each $i \in N$ implies that $K(p)=K(q)$.

Strong positive association: The choice rule $K$ is strongly positively associated if for each $p, q \in L(A)$ and each $a \in A$,

$\operatorname{low}(a, p(i)) \subseteq \operatorname{low}(a, q(i))$ for each $i \in N$ and $\left.p(i)\right|_{A-\{a\}}=\left.q(i)\right|_{A-\{a\}}$ implies that $K(q) \in\{K(p), a\}$.

Decisiveness of coalitions appear also in our setting as a powerful tool to analyze the problem at hand.

Decisiveness: At choice rule $K$, a coalition $M \subseteq N$ is said to be decisive on $(x, y) \in A \times A$, if $K(p)=x$ for each profile $p \in \bar{L}_{x}^{M} \times L_{y}^{N-M}$.

Let $D_{K}(M)=\{(x, y) \in A \times A: M$ is decisive on $(x, y)\}$. If $K$ is Paretoefficient, then it follows immediately that $I_{A} \subseteq D_{K}(M)$. It appears that the rules which simultaneously satisfy Pareto-efficiency, non-dictatorship and either strategy-proofness or Maskin monotonicity on the domains at hand are almost dictatorial.

Let $I_{A} \varsubsetneqq D \varsubsetneqq A \times A$ for some transitive relation $D$ on $A$. Define by $K_{D}$ the hierarchical choice rule corresponding to $D$ defined as follows: for every profile $p$ such that $p(1) \in L_{y}^{1}, K_{D}(p)=\operatorname{best}\left(\left.p(2)\right|_{u p(y, D)}\right)$. Because of $D$ being unequal to both $I_{A}$ and $A \times A$, it follows that $K_{D}$ is non-dictatorial. Transitivity of $D$ imposes a kind of rationality which under Pareto-efficiency ${ }^{10}$ is implied by both Maskin-monotonicity and strategy-proofness. Next, we discuss conditions that $D$ has to satisfy in order for $K_{D}$ to be a strategy-proof or Maskin monotonic social choice rule. We introduce the following definitions.

Strategy-proof admissibility: The pair $\left(D, L^{1}\right)$ is strategy-proof admissible if $I_{A} \varsubsetneqq D \varsubsetneqq A \times A, D$ is transitive and for each $(x, y) \in D$, each $R \in L_{y}^{1}$ and each $z \in u p(x, R)$, both

$$
\begin{aligned}
& (z, y) \in D \text { and } \\
& (x, z) \in D \text { whenever } L_{z}^{1} \neq \emptyset .
\end{aligned}
$$

\footnotetext{
${ }^{10}$ But independent of the actual preference domain restriction of agent 1
} 
Let strategy $\left(D, L^{1}\right)$ stands for the strategy-proof admissibility of the pair $\left(D, L^{1}\right)$.

Maskin admissibility: The pair $\left(D, L^{1}\right)$ is Maskin admissible, notation $\operatorname{Maskin}\left(D, L^{1}\right)$ if $I_{A} \varsubsetneqq D \varsubsetneqq A \times A, D$ is transitive and for all $(x, y) \in D$ all $R \in L_{y}^{1}$ all $z \in u p(x, R)$ and all $R^{\prime} \in L_{z}^{1}$ both

$$
\begin{aligned}
& (z, y) \in D \text { and } \\
& (x, z) \in D \text { whenever } \operatorname{low}(x, R) \subseteq \operatorname{low}\left(x, R^{\prime}\right) .
\end{aligned}
$$

Let Maskin $\left(D, L^{1}\right)$ stands for the Maskin admissibility of the pair $\left(D, L^{1}\right)$.

\section{Sufficient conditions, strategy-proofness and Maskin monotonicity}

The following Lemma shows that the condition of strategy-proof admissibility and Maskin admissibility are sufficient to guarantee that $K_{D}$ is strategy-proof or Maskin-monotone respectively and therewith explains the names of these two requirements on $D$ and $L^{1}$.

Lemma 1 Consider the hierarchical choice rule $K_{D}: L^{N} \rightarrow A$, corresponding to $D$. Then

1. strategy $\left(D, L^{1}\right)$ implies that $K_{D}$ is non-dictatorial, Pareto-efficient and strategy-proof;

2. $\operatorname{Maskin}\left(D, L^{1}\right)$ implies that $K_{D}$ is non-dictatorial, Pareto-efficient and Maskin-monotone.

Proof. Non-dictatorship follows in both cases because of $I_{A} \varsubsetneqq D \varsubsetneqq A \times A$. To prove Pareto-efficiency let $p$ be a profile with $p(1) \in L_{y}^{1}$. It is sufficient to prove that Pareto-efficiency is satisfied at this profile $p$. This is indeed the case whenever $K_{D}(p)=y$. So, suppose that $K_{D}(p)=\operatorname{best}\left(\left.p(2)\right|_{u p(y, D)}\right)$ and $K_{D}(p) \neq y$. Because of $\operatorname{strategy}\left(D, L^{1}\right)$ as well as $\operatorname{Maskin}\left(D, L^{1}\right)$, it follows that $z \in u p(y, D)$ if there are $x \in u p(y, D)$ and $R \in L_{y}^{1}$ with $y \ldots z \ldots x \ldots=R$. So, for all $a \in$ non-up $(y, D)$, we have $\ldots K_{D}(p) \ldots a \ldots=p(1)$ and for all $a \in$ $u p(y, D)-\left\{K_{D}(p)\right\}$, we have $\ldots K_{D}(p) \ldots a \ldots=p(2)$. So, Pareto-efficiency is satisfied at $p$. 
In order to prove (1), assume strategy $\left(D, L^{1}\right)$. Furthermore, let $p$ be as above and profile $q$ an $\{i\}$-deviation of $p$ for some agent $i$ with $q(1) \in L_{z}^{1}$ for some $z \in A$. To the contrary suppose that $\ldots K_{D}(q) \ldots K_{D}(p) \ldots=p(i)$. By the definition of $K_{D}$ it follows that $i \leqslant 2$. If $i=2$, then $p(1)=q(1)$. Consequently, $y=z$ and $K_{D}(q)=\operatorname{best}\left(\left.q(2)\right|_{u p(z, D)}\right)=\operatorname{best}\left(\left.q(2)\right|_{u p(y, D)}\right)$. So, in that case $K_{D}(q) \in u p(y, D)$. Because $K_{D}(p)=\operatorname{best}\left(\left.p(2)\right|_{u p(y, D)}\right)$, we obtain a contradiction with $\ldots K_{D}(q) \ldots K_{D}(p) \ldots=p(i)$. To conclude the proof of $(1)$, let $i=1$. Therefore, $p(2)=q(2)$. Because of $\ldots K_{D}(q) \ldots K_{D}(p) \ldots=p(1)$ and $\operatorname{strategy}\left(D, L^{1}\right)$, it follows that $K_{D}(p) \in u p\left(K_{D}(q), D\right)$. Thus, since $D$ is transitive, we conclude that $K_{D}(p) \in u p(z, D)$. But then $K_{D}(q)=$ $\operatorname{best}\left(\left.q(2)\right|_{u p(z, D)}\right)=\operatorname{best}\left(\left.p(2)\right|_{u p(z, D)}\right)$ and $\ldots K_{D}(q) \ldots K_{D}(p) \ldots=p(1)$ contradict each other. This proves strategy-proofness.

In order to prove $(2)$, assume $\operatorname{Maskin}\left(D, L^{1}\right)$ and consider $\{i\}$-deviations $p$ and $q$ as before with, in addition, $\operatorname{low}\left(K_{D}(p), p(i)\right) \subseteq l o w\left(K_{D}(p), q(i)\right)$. It is sufficient to prove that $K_{D}(p)=K_{D}(q)$. This obviously holds whenever $i \geqslant 2$. So suppose that $i=1$. Since low $\left(K_{D}(p), p(1)\right) \subseteq$ low $\left(K_{D}(p), q(1)\right)$, it follows that $z \in u p\left(K_{D}(p), p(1)\right)$. Maskin $\left(D, L^{1}\right)$, or reflexivity of $D$, or $z=y$ imply that $\left(K_{D}(p), z\right) \in D$ and $(z, y) \in D$. Furthermore, transitivity of $D$ implies that $u p(z, D) \subseteq u p(y, D)$. But because $K_{D}(p)=\operatorname{best}\left(\left.p(2)\right|_{u p(y, D)}\right)$, it follows that $K_{D}(p)=\operatorname{best}\left(\left.p(2)\right|_{u p(y, D)}\right)=\operatorname{best}\left(\left.p(2)\right|_{u p(z, D)}\right)=\operatorname{best}\left(\left.q(2)\right|_{u p(z, D)}\right)=$ $K_{D}(q)$.

A domain $L^{N}$ is called a strategy-proof possibility domain if there exist choice rules $K: L^{N} \rightarrow A$ which are simultaneously non-dictatorial, strategyproof and Pareto-efficient. Furthermore, it is called a maximal strategy-proof possibility domain if it is a strategy-proof possibility domain and there is no other strategy-proof possibility domain, say $\widehat{L}^{N}$, such that $L^{N} \varsubsetneqq \widehat{L}^{N}$. Similarly we define a Maskin-monotone possibility domain and a maximal Maskinmonotone possibility domain by replacing the condition of strategy-proofness by the condition of Maskin monotonicity in the two previous definitions.

Example 1 In case $L^{1}$ has an inseparable pair $(y, x)$ or an inseparable set $B$, it follows straightforwardly that strategy $\left(D, L^{1}\right)$, where $D=\{(x, y)\}$ or $D=B \times B$ respectively. So, the previous Lemma implies that in these situations, the hierarchical choice rule $K_{D}$ is non-dictatorial, Pareto-efficient and strategy-proof. Thus, in that case, $L^{N}$ is a strategy-proof possibility domain.

The following results logically link several of the conditions defined above. 
Theorem 1 Let $K: L^{N} \rightarrow A$ be a social choice rule. Then

1. $K$ is strategy-proof if and only if it is intermediate strategy-proof;

2. If $K$ is strategy-proof, then it is Maskin monotonic and strongly positively associated;

3. If $L^{N}$ is convex, then strategy-proofness of $K$ is equivalent to Maskin monotonicity of $K$, as well as to strong positive association of $K$.

Proof. (First part) Clearly intermediate strategy-proof ness implies strategyproofness. So, suppose $K$ is strategy-proof. Furthermore, let $p$ and $q$ be $S$-deviations for some $S \subseteq N$ such that $p(i)=R$ for some $R \in L(A)$. It is sufficient to prove that $\ldots K(q) \ldots K(p) \ldots \neq R$. Without loss of generality let $S=\{1,2, \ldots, s\}$. Take profiles $r^{0}, r^{1}, r^{2}, \ldots, r^{s} \in L^{N}$ defined for all $i \in N$ by $r^{t}(i)=p(i)$ if $i>t$, and by $r^{t}(i)=q(i)$ otherwise. So, $r^{0}=p$ and $r^{s}=q$. By strategy-proofness, it follows that $\ldots K\left(r^{t+1}\right) \ldots K\left(r^{t}\right) \ldots \neq r^{t}(t+1)=R$ for all $t \in\{0,1,2, \ldots, s-1\}$. Because of negative transitivity of $R$, it follows that $\ldots K(q) \ldots K(p) \ldots \neq R$.

(Second part) Let $p$ and $q$ be $\{i\}$-deviations in $L^{N}$ such that,

$$
\operatorname{low}(K(p), p(i)) \subseteq \operatorname{low}(K(p), q(i)) .
$$

It is sufficient to prove that $K(p)=K(q)$. By strategy-proofness, it follows that $K(q) \in \operatorname{low}(K(p), p(i))$ and $K(p) \in$ low $(K(q), q(i))$. Given our assumption, we have that $K(q) \in \operatorname{low}(K(p), q(i))$ and $K(p) \in \operatorname{low}(K(q), q(i))$. As $q(i)$ is antisymmetric we have that $K(p)=K(q)$.

In order to prove strong positive association, let $p$ and $q$ be $\{i\}$-deviations in $L^{N}$. Furthermore, let $a \in A$ be such that low $(a, p(i)) \subseteq$ low $(a, q(i))$ and $\left.p(i)\right|_{A-\{a\}}=\left.q(i)\right|_{A-\{a\}}$. It is sufficient to prove that $K(q) \in\{a, K(p)\}$. Suppose that $K(p) \neq K(q)$. Then we have to prove that $K(q)=a$. By strategyproofness, it follows that $\ldots K(p) \ldots K(q) \ldots=p(i)$ and $\ldots K(q) \ldots K(p) \ldots=q(i)$. Because $\left.p(i)\right|_{A-\{a\}}=\left.q(i)\right|_{A-\{a\}}$ and low $(a, p(i)) \subseteq$ low $(a, q(i))$, this can only hold if $K(q)=a$.

(Third part) Let $p$ and $q$ be two $\{i\}$-deviations in $L^{N}$. It is sufficient that both Maskin monotonicity and strong positive association imply that,

$$
K(q) \in \operatorname{low}(K(p), p(i)) .
$$

Because of convexity of $L^{N}$ there are $R^{0}, R^{1}, R^{2}, \ldots, R^{k} \in L^{i}$ such that $R^{0}=$ $p(i), R^{k}=q(i), R^{t} \Delta R^{t+1}=\left\{\left(x_{t}, y_{t}\right),\left(y_{t}, x_{t}\right)\right\}$ for some $x_{t}$ and $y_{t}$ in $A$, with 
$x_{t} \neq y_{t}$, for all $t \in\{0,1,2, \ldots, k-1\}$ and $\ldots y_{t} \ldots x_{t} \ldots=R^{s}$ for all $t<s \leqslant k$ and $\ldots x_{t} \ldots y_{t} \ldots=R^{s}$ for all $0 \leqslant s \leqslant t$. Now take profiles $r^{0}, r^{1}, r^{2}, \ldots, r^{k} \in L^{N}$, $\{i\}$-deviation of $p$ such that $r^{t}(i)=R^{t}$ for all $t \in\{0,1,2, \ldots, k\}$. Now if $K\left(r^{t}\right) \neq K\left(r^{t+1}\right)$ for some $t \in\{0,1,2, \ldots, k-1\}$, then Maskin monotonicity as well as strong positive association imply that $K\left(r^{t}\right)=x_{t}$ and $K\left(r^{t+1}\right)=y_{t}$. Hence $K\left(r^{t+1}\right) \in \operatorname{low}\left(K\left(r^{t}\right), p(i)\right)$. By transitivity, it follows that $K\left(r^{k}\right) \in$ low $\left(K\left(r^{0}\right), p(i)\right)$. So, $K(q) \in$ low $(K(p), p(i))$ proving that $K$ is strategy-proof.

\section{Necessary conditions for strategy-proofness or Maskin-monotonicity}

In the previous section, the conditions of strategy-proof admissibility and of Maskin admissibility appeared to be sufficient to guarantee the existence of choice rules on the restricted domain that are Pareto-efficient, non-dictatorial and strategy-proof, or Maskin monotonic, respectively. Here we prove that these two conditions are in fact also necessary.

For the rest of this section, let $K$ be a Pareto-efficient and non-dictatorial social choice rule. We will show that whenever $K$ is strategy-proof or Maskin monotonic, then $I_{A} \varsubsetneqq D_{K}(N-\{1\}) \varsubsetneqq A \times A$ is transitive and that the pair $\left(D_{K}(N-\{1\}), L^{1}\right)$ is respectively strategy-proof admissible or Maskin admissible.

To avoid needless repetitions, assume that $K$ is at least Maskin monotonic. So, cases at which the social choice rule $K$ is strategy-proof are spelled out explicitly in this section.

Lemma 2 Let $p \in L^{N}, M \subseteq N$ and $(x, y) \in A \times(A \backslash\{x\})$ such that for each agent $i \in M, x y \ldots=p(i)$ and for each agent $i \in N-M, y \ldots=p(i)$. Let $K(p)=x$. Then $(x, y) \in D_{K}(M)$.

Proof. Let $q \in L_{x}^{M} \times L_{y}^{N-M}$. It is sufficient to prove that $K(q)=x$. Let $r \in L^{N}$ be an $(N-M)$-deviation of $p$ and an $M$-deviation of $q$. Now, Paretoefficiency implies that $K(r) \in\{x, y\}$. If $K(r)=y$, then Maskin monotonicity would imply the contradiction that $K(p)=y$. Therefore, $K(r)=x$. But then Maskin monotonicity implies that $K(q)=x$.

Lemma $3 I_{A} \varsubsetneqq D_{K}(N-\{1\}) \varsubsetneqq A \times A$ and $D_{K}(N-\{1\})$ is transitive. 
Proof. (Proof of $\left.I_{A} \varsubsetneqq D_{K}(N-\{1\})\right)$ To the contrary, suppose that $I_{A}=$ $D_{K}(N-\{1\})$. We show that this leads to the contradiction that agent 1 is a dictator. Consider $R \in L^{1}$ and a profile $p \in L^{N}$, with $x y \ldots=R=p(1)$ for some $x$ and $y$. To deduce the contradiction, it is sufficient to prove that $K(p)=x$. Consider the profiles $q$ and $r$-both $N-\{1\}$-deviations of $p$-such that $q(i)=y x \ldots$ and $r(i)=y \ldots x$ for each $i \geqslant 2$. Because of Paretoefficiency, it follows that $K(q) \in\{x, y\}$. Now, by Lemma 2, it follows that $K(q) \neq y$, otherwise $(y, x) \in\left(D_{K}(N-\{1\})-I_{A}\right)$. But then $K(q)=x$ and $(x, y) \in D_{K}(\{1\})$. So, $K(r)=x$. By Maskin monotonicity, we obtain that $K(p)=x$.

(Proof of $\left.D_{K}(N-\{1\}) \varsubsetneqq A \times A\right)$ To the contrary suppose that $D_{K}(N-$ $\{1\})=A \times A$. We show that this leads to the contradiction that $K$ is dictatorial. For $R \in L^{1}$, consider the social choice rule $K^{R}$ defined by $K^{R}(p)=K(R, p)$ for each profile $p \in L(A)^{N-\{1\}}$. It is clear that $K^{R}$ is surjective-and even unanimous-and Maskin monotonic. By Theorem 1, it is strategy-proof. Hence, by the Gibbard and Satterthwaite Theorem, it follows that $K^{R}(p)$ is dictatorial, say by agent $i_{R} \geqslant 2$.

Consider two preferences $R$ and $R^{\prime}$ in $L^{1}$. In order to prove that $K$ is dictatorial, it is sufficient to show that $i_{R}=i_{R^{\prime}}$. To the contrary, suppose that $i_{R} \neq i_{R^{\prime}}$. We deduce a contradiction and are done. Obviously there are different alternatives $x, y, z_{1}, z_{2}, \ldots z_{k-1}$ and $z_{k}$, where $k$ may be zero, such that $z_{1} z_{2} \ldots z_{k} x \ldots=R$ and $z_{1} z_{2} \ldots z_{k} y \ldots=R^{\prime}$. So $x$ and $y$ are the first alternatives on which the preferences $R$ and $R^{\prime}$ differ. Consider profiles $p$ and $q$ which are $\{1\}$-deviations such that $p(1)=R, q(1)=R^{\prime}, p\left(i_{R}\right)=q\left(i_{R}\right)=y \ldots$ and $p\left(i_{R^{\prime}}\right)=q\left(i_{R^{\prime}}\right)=x \ldots$. Then since $i_{R}$ is a dictator at $K^{R}$, it follows that $K(p)=y$ and because $i_{R^{\prime}}$ is a dictator at $K^{R^{\prime}}$, it follows that $K(q)=x$. Finally, because $\operatorname{low}(y, R) \subseteq \operatorname{low}\left(y, R^{\prime}\right)$, we have a contradiction with Maskin monotonicity of $K$.

(Proof of transitivity) Let $(x, y),(y, z) \in D_{K}(N-\{1\})$. It is sufficient to prove that $(x, z) \in D_{K}(N-\{1\})$. This is trivially the case when $x, y$ and $z$ are not three different alternatives. So, let these three alternatives be different. Let $R=z \ldots \in L^{1}$ arbitrary. Consider $p \in L^{N}$ such that $p(1)=R$ and $p(i)=x z \ldots$ for each $i \in N-\{1\}$. By Lemma 2, it is sufficient to prove that $K(p)=x$. Consider $(N-\{1\})$-deviation $q$ of $p$ such that $x y z \ldots=q(i)$ for all $i \in N-\{1\}$. If $K(q)=y$, then Maskin monotonicity would yield $K(r)=y$-where $r$ is the $\{1\}$-deviation of $q$ with $r(1)=y \ldots$-which contradicts $(x, y) \in D(N-\{1\})$. If $K(q)=z$, then Maskin monotonicity would yield $K(u)=z$, where $u$ is a $(N-\{1\})$-deviation of $q$, with $y z \ldots=u(i)$ for all 
$i \in N-\{1\}$, which contradicts $(y, z) \in D(N-\{1\})$. Therefore $K(q)=x$ and Maskin monotonicity implies that $K(p)=x$.

Lemma 4 Let $x, y$ and $z$ be different alternatives and $R \in L^{1}$ such that $y \ldots z \ldots \ldots x \ldots=R$. Let $(x, y) \in D_{K}(N-\{1\})$. Then

1. $(z, y) \in D_{K}(N-\{1\})$;

2. $(x, z) \in D_{K}(N-\{1\})$ if $K$ is strategy-proof and $L_{z}^{1} \neq \emptyset$;

3. $(x, z) \in D_{K}(N-\{1\})$ if there are $R^{\prime} \in L_{z}^{1}$ such that $\operatorname{low}(x, R) \subseteq$ low $\left(x, R^{\prime}\right)$.

Proof. (Proof of 1) Let $q \in L^{N}$ such that $q(1)=R$ and $z y \ldots=q(i)$ for all $i \in N-\{1\}$. By Lemma 2 it is sufficient to prove that $K(q)=z$. Consider $p$ and $r$, two $(N-\{1\})$-deviations of $q$, such that $x z y \ldots=p(i)$ and $z x y \ldots=r(i)$ for all $i \in N-\{1\}$. Because of $(x, y) \in D(N-\{1\})$, it follows that $K(p)=x$. Pareto-efficiency implies that $K(r) \in\{y, z\}$. If $K(r)=y$, then Maskin monotonicity would yield the contradiction that $K(p)=y$. Therefore, $K(r)=z$ and Maskin monotonicity implies that $K(q)=z$.

(Proof of 2) Suppose $K$ is strategy-proof and $z \ldots=R^{\prime} \in L_{z}^{1} \neq \emptyset$. Let $q \in L^{N}$ such that $q(1)=R^{\prime}$ and $x z \ldots=q(i)$ for all $i \in N-\{1\}$. By Lemma 2 , it is sufficient to prove that $K(q)=x$. Consider $(N-\{1\})$-deviations $p$ of $q$, with $p(1)=R$. Because of $(x, y) \in D(N-\{1\})$, it follows that $K(p)=x$. Pareto-efficiency implies that $K(q) \in\{x, z\}$. Now considering $p$ and $q$, it follows that $K(q)=z$ would violate strategy-proofness. In consequence, $K(q)=x$.

(Proof of 3) Suppose $R^{\prime} \in L_{z}^{1}$ such that $\operatorname{low}(x, R) \subseteq \operatorname{low}\left(x, R^{\prime}\right)$. Let $q \in L^{N}$ be such that $q(1)=R^{\prime}$ and $x z \ldots=q(i)$ for all $i \in N-\{1\}$. By Lemma 2 , it is sufficient to prove that $K(q)=x$. Consider $(N-\{1\})$-deviations $p$ of $q$, with $p(1)=R$. Because of $(x, y) \in D(N-\{1\})$, it follows that $K(p)=x$. Next, because low $(x, R) \subseteq \operatorname{low}\left(x, R^{\prime}\right)$ and Maskin monotonicity, we have that $K(q)=x$.

Combining Lemma's 1, 2, 3 and 4 yields the following corollaries.

Corollary 1 If $L^{N}$ is a Maskin monotonic possibility domain, then $\left(D_{K}(N-\right.$ $\left.\{1\}), L^{1}\right)$ is Maskin admissible;

Corollary 2 If $L^{N}$ is a strategy-proof possibility domain, then $\left(D_{K}(N-\right.$ $\left.\{1\}), L^{1}\right)$ is strategy-proof admissible. 


\section{Maximal domains for strategy-proofness}

Corollary 2 characterizes strategy-proof possibility domains in terms of a set of pairs of alternatives on which the coalition of agents whose sets of preferences are not restricted are decisive. Consider a Pareto-efficient, strategy-proof and non-dictatorial social choice rule $K$, such that for all strategy-proof possibility domains $\widehat{L}^{N}$ with $L^{N} \subseteq \widehat{L}^{N}$, we have that $L^{N}=\widehat{L}^{N}$. So, $L^{N}$ is a maximal strategy-proof possibility domain. We shall prove in Theorem 2 that the conditions on $D_{K}(N-\{1\})$ under this maximality property yield that $L^{1}$ either has one inseparable pair or one inseparable set. Furthermore, we deduce that these separabilities are not only necessary but also sufficient. The following Lemmas are needed for the proof of Theorem 2 .

Lemma 5 Let $x \in A$. Then $L_{x}^{1} \neq \emptyset$.

Proof. To the contrary let $L_{x}^{1}=\emptyset$. Take $\widehat{L}^{1}=L^{1} \cup\{R \in L(A): x y \ldots=R\}$ for some fixed $y \in A-\{x\}$. Clearly, by taking $\widehat{L}^{i}=L(A)$ for $i>1$, we obtain that $L^{N} \varsubsetneqq \widehat{L}^{N}$. By lemma 1, the latter is a strategy-proof possibility domain. This yields a contradiction with $L^{N}$ being maximal.

Intuitively it is reasonable that the smaller the set of decisive pairs of $N-\{1\}$, the larger $L^{1}$ can be taken. The following Lemma shows that we may shrink the set of decisive pairs of $N-\{1\}$.

Lemma 6 Let $\left(x^{*}, y^{*}\right) \in D_{K}(N-\{1\})$, with $x^{*} \neq y^{*}$.

Take,

$$
\begin{gathered}
Y=\left\{a \in A: a=y^{*} \text { or }\left(a, y^{*}\right),\left(y^{*}, a\right) \in D_{K}(N-\{1\})\right\} \text { and, } \\
Z=\left\{a \in A:\left(a, y^{*}\right) \in D_{K}(N-\{1\}) \text { and }\left(y^{*}, a\right) \notin D_{K}(N-\{1\})\right\} . \\
\text { Let } D^{*}=\left[(Z \times Z) \cup(Z \times Y) \cup(Y \times Y) \cup I_{A}\right] \cap D_{K}(N-\{1\}) .
\end{gathered}
$$

Then strategy $\left(D^{*}, L^{1}\right)$. Furthermore, $\left[(Z \times Y) \cup(Y \times Y) \cup I_{A}\right] \subseteq D_{K}(N-$ $\{1\})$.

Proof. By definition $\left(x^{*}, y^{*}\right) \in D^{*}-I_{A}$ and $D^{*} \subseteq D_{K}(N-\{1\})$. So, $I_{A} \varsubsetneqq D^{*} \varsubsetneqq A \times A$. Because of transitivity of $D_{K}(N-\{1\})$ and $[(Z \times Z) \cup$ $\left.(Z \times Y) \cup(Y \times Y) \cup I_{A}\right]$ it follows that $D^{*}$ is transitive. Let $x, y, z \in A$, with $\#\{x, y, z\}=3$. Let $(x, y) \in D^{*}$. Let $y \ldots z \ldots x \ldots=R$ in $L^{1}$. It is sufficient to 
prove that $(z, y) \in D^{*}$ and $(x, z) \in D^{*}$. Because of strategy $\left(D_{K}(N-\{1\}), L^{1}\right)$ and Lemma 5 , it follows that $(x, z),(z, y) \in D_{K}(N-\{1\})$. Moreover, by transitivity of $D_{K}(N-\{1\})$ and the definition of $Y$ and $Z$, it follows that $D_{K}(N-\{1\}) \cap(Y \times Z)=\emptyset$. Thus, it is sufficient to prove that $z \in Y \cup Z$. Because of $y \in Y \cup Z$, it follows that $\left(y, y^{*}\right) \in D_{K}(N-\{1\})$. However, $(z, y) \in D_{K}(N-\{1\})$ and transitivity of $D_{K}(N-\{1\})$ together imply that $\left(z, y^{*}\right) \in D_{K}(N-\{1\})$. Therefore, $z \in Y \cup Z$.

The furthermore part follows by transitivity of $D_{K}(N-\{1\})$ and the definition of $Y$ and $Z$.

Lemma 7 There are disjoint subsets $Y$ and $Z$ of $A$ such that

1. $Y$ is non-empty, $Y \cup Z \neq A$, and $\#(Y \cup Z) \geqslant 2$;

2. $I_{A} \varsubsetneqq\left[((Y \cup Z) \times Y) \cup I_{A}\right] \subseteq D_{K}(N-\{1\}) \varsubsetneqq A \times A$ and

3. $\operatorname{strategy}\left(((Y \cup Z) \times Y) \cup I_{A}, L^{1}\right)$.

Proof. In Lemma 6, we proved that there are disjoint $Y$ and $Z$ such that strategy $\left(\left[(Z \times Z) \cup(Z \times Y) \cup(Y \times Y) \cup I_{A}\right] \cap D_{K}(N-\{1\}), L^{1}\right)$. Because of the transitivity of $D_{K}(N-\{1\})$, we may take $\left(x^{*}, y^{*}\right) \in D_{K}(N-\{1\})$ such that $x^{*} \neq y^{*}$ and $(Z \times Z) \cap D_{K}(N-\{1\}) \subseteq I_{A}$. By the furthermore part of the previous Lemma 6, we obtain that $(Y \cup Z) \times Y \subseteq D_{K}(N-\{1\})$. Hence, it follows that strategy $\left(((Y \cup Z) \times Y) \cup I_{A}, L^{1}\right)$. The inclusions at (2) follow readily.

Also, by definition, $Y$ is non-empty and because of $x^{*}, y^{*} \in Y \cup Z$, with $x^{*} \neq y^{*}$, we have $\#(Y \cup Z) \geqslant 2$.

It remains to prove that $Y \cup Z \neq A$. In order to do so, suppose that $Y \cup Z=A$. We prove that we may take $Z=\emptyset$ and that $Y \neq A$. Consider $y \in Y$ and $R=y \ldots a$ in $L^{1}$ where $a \in A$. Because of strategy $(((Y \cup Z) \times Y) \cup$ $\left.I_{A}, L^{1}\right)$ and $(a, y) \in((Y \cup Z) \times Y)$, it follows for each $b \in A-\{y, a\}$ that $(a, b) \in((Y \cup Z) \times Y)$. Hence, $b \in Y$ for each $b \in A-\{a, y\}$. Next, because $\left[((Y \cup Z) \times Y) \cup I_{A}\right] \varsubsetneqq A \times A$, it follows that $Z \neq \emptyset$. But then $Z=\{a\}$. As the previous holds for every $a^{\prime} \in A$ for which there are $y \in Y, R \in L_{y}^{1}$ with $y \ldots a^{\prime}=R$ and $Z$ is a singleton, it follows that for each $y \in Y$ and each $R \in L_{y}^{1}$ that $y \ldots a=R$. Hence, strategy $\left((Y \times Y) \cup I_{A}, L^{1}\right)$ and as $Z$ is a singleton $Y \neq A$. By taking $Z=\emptyset$ this shows the existence of such $Y$ and $Z$. 
Theorem 2 Consider $L^{N}$ with $L^{i}=L(A)$ for $i \geqslant 2$. Then $L^{N}$ is a maximal strategy-proof possibility domain if and only if one of the following two holds:

1. There are $a, b \in A$ with $a \neq b$ and $L^{1}=V$ where $V=\{R \in L(A)$ : if $R \in L_{a}^{1}$, then $\left.a b \ldots=R\right\}$ which means that $L^{1}$ has an inseparable pair $(a, b)$;

2. There is a non-trivial subset $Y$ of $A$, with $\# Y \geqslant 3$, such that $L^{1}=W$ where $W=\left\{R \in L(A):\right.$ if $R \in L_{y}^{1}$ for some $y \in Y$, then for all $a \in Y$ and all $b \in A-Y$ we have $\ldots a \ldots b \ldots=R\}$ which means that $L^{1}$ has an inseparable set $Y$.

Proof. (Only-if-part) Suppose $L^{1}$ is a maximal strategy-proof possibility domain. By the previous Lemma there are disjoint subsets $Y$ and $Z$ of $A$ such that

1. $Y$ is non-empty, $Y \cup Z \neq A$, and $\#(Y \cup Z) \geqslant 2$;

2. $I_{A} \varsubsetneqq\left[((Y \cup Z) \times Y) \cup I_{A}\right] \varsubsetneqq A \times A$ and

3. $\operatorname{strategy}\left(((Y \cup Z) \times Y) \cup I_{A}, L^{1}\right)$.

Consider $y \in Y$ and $z, t \in Y \cup Z$. Take $R \in L_{y}^{1}$ which exist by Lemma 5 and assume without loss of generality that $y \ldots t \ldots z \ldots=R$. Then by strat$\operatorname{egy}\left(((Y \cup Z) \times Y) \cup I_{A}, L^{1}\right)$, it follows that $(z, t) \in(Y \cup Z) \times Y$. Hence, $t \in Y$. So, $\# Z \leqslant 1$. Moreover, if $z \in Z$, then for all $t \in Y-\{y\}$, we have $y \ldots t \ldots z \ldots=R$. Because $y$ is chosen arbitrarily, this means that strat$\operatorname{egy}\left((Y \times Y) \cup I_{A}, L^{1}\right)$ or $\# Y=\# Z=1$. Suppose $Y=\{b\}$ and $Z=\{a\}$. Then strategy $\left(((Y \cup Z) \times Y) \cup I_{A}, L^{1}\right)$ implies that $L^{1}$ has an inseparable pair $(a, b)$. Consider the set $V$. We shall prove $L^{1}=V$. Because obviously strat$\operatorname{egy}\left(((Y \cup Z) \times Y) \cup I_{A}, V\right)$ and therefore strategy $\left(((Y \cup Z) \times Y) \cup I_{A}, L^{1} \cup V\right)$ it follows by the maximality of $L^{1}$ that $V \subseteq L^{1}$. Now $V$ is defined such that it contains all sets of preferences which have an inseparable pair $(a, b)$. Therefore $L^{1} \subseteq V$. So, $L^{1}=V$. Now suppose $\operatorname{strategy}\left((Y \times Y) \cup I_{A}, L^{1}\right)$. Then it follows that $L^{1}$ has an inseparable set $Y$. Consider the set $W$. By proving that $L^{1}=W$, we end the proof of the only-if-part. Because obviously strat$\operatorname{egy}\left((Y \times Y) \cup I_{A}, W\right)$ and therefore obviously strategy $\left((Y \times Y) \cup I_{A}, L^{1} \cup W\right)$, it follows by the maximality of $L^{1}$ that $W \subseteq L^{1}$. But $W$ is defined such that is contains all sets of preferences which have an inseparable set $Y$. Therefore $L^{1} \subseteq V$. So, $L^{1}=W$. 
(If-part) By example 1 it is clear that if $L^{1}$ equals either $V$ or $W$, then $L^{N}$ is a strategy-proof possibility domain. It remains to prove the maximality of it. Suppose $L^{i} \subseteq \widehat{L}^{i}$ for all agents $i$ and $\widehat{L}^{N}$ is a maximal strategy-proof possibility domain. It is sufficient to prove that $L^{N}=\widehat{L}^{N}$. By the only-if-part, it follows that $\widehat{L}^{1}$ has either an inseparable set say $\widehat{Y}$ or an inseparable pair say $(\widehat{a}, \widehat{b})$. Because $L^{1} \subseteq \widehat{L}^{1}$ and is such that it contains all sets of preferences which either have an inseparable set $Y$ or an inseparable pair $(a, b)$, it follows that the inseparable sets or pairs are equal and that $L^{1}=\widehat{L}^{1}$.

\section{Maximal domains for Maskin-monotonicity}

In this section we characterize the maximal Maskin monotone possibility domains for the case that precisely one agent's set of preferences is restricted. Theorem 3 spells out a characterization of Maskin monotone possibility domains in terms of a set of pairs of alternatives on which the coalition of agents whom sets of preferences are not restricted are decisive.

Remark 1 Consider the Lemma's 5', 6' and 7' obtained from Lemma's 5, 6 and 7 by replacing the word "strategy" by the word "Maskin" respectively - The proofs of these Lemma's follow likewise by the same substitution in the proofs of the original Lemma's. To avoid obvious repetitions neither the Lemma's 5', 6' and '7' nor their proofs are written out here.

Theorem 3 Let $N=\{1, \ldots, n\}$ and $n \geqslant 2$. Let $L^{i}=L(A)$ for all $i \geqslant 2$. Then $L^{N}$ is a maximal Maskin monotone possibility domain if and only if there are disjoint subsets $Y$ and $Z$ of $A$ and an asymmetric and transitive relation $P$ on $A$ such that

a $Y$ is non-empty, $Y \cup Z \neq A, \#(Y \cup Z) \geqslant 2$ and $[(Y \cup Z) \times(A-(Y \cup Z))] \subseteq$ $P \subseteq[(Y \cup Z) \times(A-Y)]$

$\mathbf{b}$ if $L^{1}$ has a separable pair $(b, a)$ then $Y=\{b\}$ and $Z=\{a\}$;

c there is no partition $X_{1}, X_{2}$ of $Y \cup Z$ with $\# X_{1} \geqslant 2$ and $X_{1} \times X_{2} \subseteq P$ and

d $L^{1}=V$ where $V=\{R \in L(A)$ :

1. if $y \ldots=R$ for some $y \in Y$, then $P \subseteq R$; 
2. if $z \ldots=R$ for some $z \in Z$, then there are $t \in A-(Y \cup Z)$ such that $[($ non-up $(z, P)-\{z\}) \cap(Y \cup Z)] \subseteq \operatorname{low}(t, R)\}$.

Proof. (Only-if-part) Let $L^{N}$ be a maximal Maskin monotone possibility domain. We will show the existence of sets $Y, Z$ and relation $P$ satisfying the conditions formulated in the theorem. By Lemma 7' we have $Y, Z \subseteq A$, where $Y \cap Z=\emptyset, Y \neq \emptyset, Y \cup Z \neq A$ and Maskin $\left(((Y \cup Z) \times Y) \cup I_{A}, L^{1}\right)$. For different alternatives $a$ and $b$, define $(a, b) \in P$ if for all $R \in L_{y}^{1}$ and all $y \in Y$ we have that $\ldots a \ldots b \ldots=R$. By definition, $P$ is asymmetric and because the preferences in $L^{1}$ are transitive, $P$ is transitive. Because of Maskin $(((Y \cup Z) \times$ $\left.Y) \cup I_{A}, L^{1}\right)$, it follows that $[(Y \cup Z) \times(A-(Y \cup Z))] \subseteq P$. Because of the definition of $P$ and Lemma 5 it follows that $P \subseteq[(Y \cup Z) \times(A-Y)]$. This proves part (a).

Claim 1 Maskin $\left(((Y \cup Z) \times Y) \cup I_{A}, V\right)$.

We have to prove the following implications for $x, y, z \in A$ and $R, R^{\prime} \in V$.

1. If $(x, y) \in(Y \cup Z) \times Y$ and $y \ldots z \ldots x \ldots=R$, then $(z, y) \in(Y \cup Z) \times Y$;

2. If $(x, y) \in(Y \cup Z) \times Y, y \ldots z \ldots x \ldots=R, z \ldots=R^{\prime}$ and $\operatorname{low}(x, R) \subseteq$ low $\left(x, R^{\prime}\right)$, then $(x, z) \in(Y \cup Z) \times Y$.

The proof of the first implication follows immediately from the first condition in the definition of $V$ and $[(Y \cup Z) \times(A-(Y \cup Z))] \subseteq P$. To prove the second implication, let $x, y, z, R$ and $R^{\prime}$ be as in the premises of implication 2. It is sufficient to prove that $\operatorname{low}(x, R) \nsubseteq \operatorname{low}\left(x, R^{\prime}\right)$ or $(x, z) \in(Y \cup Z) \times Y$. Clearly by implication 1 , it follows that $z \in(Y \cup Z)$. If $z \in Y$, then evidently $(x, z) \in(Y \cup Z) \times Y$. So, suppose $z \notin Y$, which implies that $z \in Z$. Now because of $P \subseteq R$ and the definition of $P$, it follows that $(x, z) \notin P$. Of course $x \neq z$. So, there are $t \in A-(Y \cup Z)$ such that $x \in l o w\left(t, R^{\prime}\right)$. Now, since $t \in A-(Y \cup Z)$, it follows that $y \ldots x \ldots t \ldots=R$. Thus, $\operatorname{low}(x, R) \nsubseteq$ low $\left(x, R^{\prime}\right)$. This proves the second implication and ends the proof of the claim.

Next we prove part (b). Let $L^{1}$ have an inseparable pair $(b, a)$. In view of Example 1, Theorem 1 and $L^{1}$ being a maximal Maskin monotone possibility domain it follows for all $x \in A-\{b\}$ that $L_{x}(A) \subseteq L^{1}$ and $L_{b}^{1}=\{R \in L(A)$ : $b a \ldots=R\}$. So, for $(x, y) \neq(a, b)$, with $x \neq y$, there are $R \in L^{1}$ such that $y \ldots x=R$. Now, $(x, y) \in(Y \cup Z) \times Y$ would, by Maskin $\left(((Y \cup Z) \times Y) \cup I_{A}, L^{1}\right)$, 
imply that $\{x\} \times A \subseteq((Y \cup Z) \times Y)$ and therewith the contradiction $A \subseteq Y$. In consequence, $((Y \cup Z) \times Y)=\{(a, b)\}$. Hence, $\{a\}=Z$ and $\{b\}=Y$. This completes the proof of part (b).

In order to prove (c) let $X_{1}$ and $X_{2}$ be a partition of $Y \cup Z$ with $\# X_{1} \geqslant 2$ and $X_{1} \times X_{2} \subseteq P$. It is sufficient to prove that this contradicts the maximality of $L^{N}$. Then $I_{A} \cup\left[X_{1} \times\left(Y \cap X_{1}\right)\right] \varsubsetneqq A \times A$ and $I_{A} \cup\left[X_{1} \times\left(Y \cap X_{1}\right)\right]$ is transitive.Bythe definition of $P$ it follows that $X_{1} \cap Y \neq \emptyset$. Consider $y \in$ $X_{1} \cap Y$ and $x \in X_{2}$. Then $(x, y) \in(Y \cup Z) \times Y$. Let $R \in L_{y}^{1}$ and let $R^{\prime} \in L(A)$ be such that $\left.R\right|_{A-\{x\}}=\left.R^{\prime}\right|_{A-\{x\}}$ and $y \ldots x=R^{\prime}$. Then $R^{\prime} \in L^{1}$ and $\operatorname{Maskin}\left(((Y \cup Z) \times Y) \cup I_{A}, L^{1}\right)$ would imply $\{x\} \times A \subseteq((Y \cup Z) \times Y)$ which then contradicts $Y \cup Z \neq A$. So, $R^{\prime} \notin L^{1}$. But obviously Maskin $\left(\left(X_{1} \times\right.\right.$ $\left.\left.\left(Y \cap X_{1}\right)\right) \cup I_{A}, L^{1} \cup\left\{R^{\prime}\right\}\right)$ which contradicts the maximality of $L^{N}$. Next we prove $(d)$.

First we prove $L^{1} \subseteq V$. Let $R \in L^{1}$. Then (1) follows because of the definition of $P$. In order to show that also (2) is satisfied let $z \ldots=R$. Let $T=$ (non-up $(z, P)-\{z\}) \cap(Y \cup Z)$. Take $x=\operatorname{best}\left(\left.R\right|_{T}\right)$. So $x \in T$ and for some $y \in Y$ there are $R^{\prime}$ in $L^{1}$ with $y \ldots z \ldots x \ldots=R^{\prime}$. Because of $z \in Z$, it follows that $(x, z) \notin((Y \cup Z) \times Y) \cup I_{A}$. Therefore Maskin $\left(((Y \cup Z) \times Y) \cup I_{A}, L^{1}\right)$ implies low $\left(x, R^{\prime}\right) \nsubseteq \operatorname{low}(x, R)$. So, there are $t \in \operatorname{low}\left(x, R^{\prime}\right)$ and $x \in \operatorname{low}(t, R)$. Because of the definition of $x$ and the fact that $x \in \operatorname{low}(t, R)$, it follows that $t \notin T$. But then, because $t \in \operatorname{low}\left(x, R^{\prime}\right)$, it follows that $t \in$ non$u p(z, P)-\{z\}$. Now, because $t \notin T$, it implies that $t \notin(Y \cup Z)$. So, $t \in A-(Y \cup Z)$ and $T \subseteq$ low $(t, R)$ which in turn yields (2).

Next we prove that $\bar{V} \subseteq L^{1}$. Because of $L^{1} \subseteq V$ and the maximality of $L^{1}$, it is sufficient to prove that $V$ is a Maskin monotone possibility domain. So, by Corollary 1 it is sufficient to prove that Maskin $\left(((Y \cup Z) \times Y) \cup I_{A}, V\right)$, which follows by the previous claim. This completes the proof of part (d) and herewith the only if part.

(If-part) Let $Y, Z, P$ and $V$ as formulated in the if part of the theorem. Let $L^{1}=V$. It is sufficient to prove that $L^{N}$ is a Maskin monotone possibility domain and that for all Maskin possibility domains $\widehat{L}^{N}$ with $V \subseteq \widehat{L}^{1}$ and $\widehat{L}^{i}=L(A)$, we have that $V=\widehat{L}^{1}$. Note that $P=\{(a, b) \in A \times A: \bar{a} \neq b$ and for all $y \in Y$ and all $\left.R \in L_{y}^{1} \ldots a \ldots b \ldots=R\right\}$. By Claim 1 we have Maskin $(((Y \cup$ $\left.Z) \times Y) \cup I_{A}, L^{1}\right)$. Setting $D$ of Lemma 1 equal to $((Y \cup Z) \times Y) \cup I_{A}$, it follows that $L^{N}$ is a Maskin monotone possibility domain. Therefore it is sufficient to prove that there are no $\emptyset \neq D \varsubsetneqq A \times A$ and $V \varsubsetneqq W \subseteq L(A)$ with $\operatorname{Maskin}(D, W)$. To the contrary, let there be such $D$ and $W$. Without loss of generality we may assume that $W$ is a maximal Maskin monotone possibility 
domain. By the only-if-part, we may assume the existence of disjoint $Y^{\prime}$, $Z^{\prime} \subseteq A$ with $Y^{\prime} \neq \emptyset, Y^{\prime} \cup Z^{\prime} \neq A$, an asymmetric and transitive relation $P^{\prime}$ on $A$ with $\left[\left(Y^{\prime} \cup Z^{\prime}\right) \times\left(A-\left(Y^{\prime} \cup Z^{\prime}\right)\right)\right] \subseteq P^{\prime} \subseteq\left[\left(Y^{\prime} \cup Z^{\prime}\right) \times\left(A-Y^{\prime}\right)\right]$, $\#\left(Y^{\prime} \cup Z^{\prime}\right) \geqslant 2$, if $L^{1}$ has a separable pair $(b, a)$ then $Y^{\prime}=\{b\}$ and $Z^{\prime}=\{a\}$, there is no partition $X_{1}^{\prime}, X_{2}^{\prime}$ of $Y^{\prime} \cup Z^{\prime}$ with $\# X_{1}^{\prime} \geqslant 2, X_{1}^{\prime} \times X_{2}^{\prime} \subseteq P^{\prime}$ and $W=\{R \in L(A)$ :

1. if $y \ldots=R$ for some $y \in Y^{\prime}$, then $P^{\prime} \subseteq R$;

2. if $z \ldots=R$ for some $z \in Z^{\prime}$, then there are $t^{\prime} \in A-\left(Y^{\prime} \cup Z^{\prime}\right)$ such that for all $x^{\prime} \in Y^{\prime} \cup Z^{\prime}$, with $x^{\prime} \neq z$ and $\left.\left(x^{\prime}, z\right) \notin P^{\prime}, x^{\prime} \in l o w\left(t^{\prime}, R\right)\right\}$.

Now by Claim 1 it follows that Maskin $\left(\left(\left(Y^{\prime} \cup Z^{\prime}\right) \times Y^{\prime}\right) \cup I_{A}, W\right)$. Because of $V \subseteq W$, this implies Maskin $\left(\left(\left(Y^{\prime} \cup Z^{\prime}\right) \times Y^{\prime}\right) \cup I_{A}, V\right)$. Next we prove that $\left(\left(\left(Y^{\prime} \cup Z^{\prime}\right) \times Y^{\prime}\right) \cup I_{A}\right)=\left(((Y \cup Z) \times Y) \cup I_{A}\right)$.

First, we prove that $\left(\left(\left(Y^{\prime} \cup Z^{\prime}\right) \times Y^{\prime}\right) \cup I_{A}\right) \subseteq\left(((Y \cup Z) \times Y) \cup I_{A}\right)$. It is sufficient to prove that $Y^{\prime} \cup Z^{\prime} \subseteq Y \cup Z$ and that $Y^{\prime} \subseteq Y$. In order to prove the former let $x \in A-(Y \cup Z)$. It is sufficient to show that $x \notin Y^{\prime} \cup Z^{\prime}$. Clearly by the definition of $V$ it follows that $L_{x}(A)=V_{x}$. Because of $V \subseteq W$, it follows that $V_{x} \subseteq W_{x}$. Hence, $L_{x}(A)=W_{x}$. Suppose $x \in Y^{\prime}$. Then there are $z \in\left(Y^{\prime} \cup Z^{\prime}\right)-\{x\}$ and $R \in L_{x}(A)=W_{x}$ such that $x \ldots z=R$. Now by the assumptions on $Y^{\prime}, Z^{\prime}, P^{\prime}$ and $W$ we have that $\left(Y^{\prime} \cup Z^{\prime}\right) \times\left(A-\left(Y^{\prime} \cup Z^{\prime}\right)\right) \subseteq P \subseteq R$. As $x \ldots z=R, x \notin Y^{\prime}$ and $z \in Y^{\prime} \cup Z^{\prime}$ this can only hold if $A=Y^{\prime} \cup Z^{\prime}$ which contradicts our assumptions on $Y^{\prime}, Z^{\prime}, P^{\prime}$ and $W$. Suppose $x \in Z^{\prime}$. Then there are $R \in L_{x}(A)=W_{x}$ such that $\left(Y^{\prime} \cup Z^{\prime}\right) \times\left(A-\left(Y^{\prime} \cup Z^{\prime}\right)\right) \subseteq R$. Now as there are $t \in A-\left(Y^{\prime} \cup Z^{\prime}\right)$ such that $\left((\right.$ non-up $\left.(z, P)-\{z\}) \cap\left(Y^{\prime} \cup Z^{\prime}\right)\right) \subseteq$ low $(t, R)$ and there are $y \in Y^{\prime}$ which clearly are in $\left((\right.$ non-up $\left.(x, P)-\{x\}) \cap\left(Y^{\prime} \cup Z^{\prime}\right)\right)$ we have a contradiction. This proves $Y^{\prime} \cup Z^{\prime} \subseteq Y \cup Z$.

In order to prove $Y^{\prime} \subseteq Y$ let $y \in Y^{\prime}$. To the contrary assume $y \notin Y$. Then by the inclusion $Y^{\prime} \cup Z^{\prime} \subseteq Y \cup Z$ we may conclude that $y \in Z$ and that there are $t \in(A-(Y \cup Z)) \subseteq\left(A-\left(Y^{\prime} \cup Z^{\prime}\right)\right)$. Furthermore, there are $R \in V_{y}$ with $y t \ldots=R$. As $V \subseteq W$, it follows that $R \in W_{y}$ where $y \in Y^{\prime}$. But $y t \ldots=R$ with $t \in A-\left(Y^{\prime} \cup Z^{\prime}\right)$ contradicts $\left[\left(Y^{\prime} \cup Z^{\prime}\right) \times\left(A-\left(Y^{\prime} \cup Z^{\prime}\right)\right)\right] \subseteq P \subseteq R$. Hence, $Y^{\prime} \subseteq Y$.

Next we prove $\left(((Y \cup Z) \times Y) \cup I_{A}\right) \subseteq\left(\left(\left(Y^{\prime} \cup Z^{\prime}\right) \times Y^{\prime}\right) \cup I_{A}\right)$. By the proof of the previous inclusion, we may assume that both $\left(Y^{\prime} \cup Z^{\prime}\right) \subseteq(Y \cup Z)$ and $Y^{\prime} \subseteq Y$. 
First we show that $Y=Y^{\prime}$. To the contrary let $b \in Y-Y^{\prime}$. Now for all $c \in Y^{\prime} \subseteq Y$, there are preferences $R$ and $R^{\prime}$ in $V$ such that $c b \ldots=R$ and $b c \ldots=R^{\prime}$. If $b \notin\left(Y^{\prime} \cup Z^{\prime}\right)$, then $R \in V \subseteq W$ violates condition 1 of $W$. So, $b \in\left(Y^{\prime} \cup Z^{\prime}\right)$ which means that $b \in Z^{\prime}$. Because of $R \in W$, it follows that $(x, b) \notin P^{\prime}$ for all $x \in A-\{c, b\}$. Now, because $R^{\prime} \in W$,in view of condition 2 of $W$ it follows that $(c, b) \in P^{\prime}$. Note that for all $x \in Y-\{b, c\}$, there are preferences $R^{\prime \prime}=x b c \ldots$ in $V \subseteq W$. So, because of $(c, b) \in P^{\prime}$, it follows that $Y^{\prime}=\{c\}$. Since $\{c, b\} \subseteq Y$, it follows that $(c, b)$ is not an inseparable pair in $V$. Therefore, there are $x \in A-\{c, b\}$ and preferences $R^{\prime \prime \prime}=c x \ldots b \ldots$ in $V \subseteq W$. Then Maskin $\left(\left(\left(Y^{\prime} \cup Z^{\prime}\right) \times Y^{\prime}\right) \cup I_{A}, W\right)$ implies $x \in\left(Y^{\prime} \cup Z^{\prime}\right)$. As $Y^{\prime}=\{c\}$ this yields $x \in Z^{\prime}$. Because of $R^{\prime \prime \prime} \in V$ and $c \in Y$, it follows that $(y, x) \notin P$ for all $y \in A-\{c, x\}$. But then, there are preferences $R^{\prime \prime \prime \prime}=b c x \ldots$ in $V \subseteq W$. Note that although $b, x \in Z^{\prime}$ and $(x, b) \notin$ $P^{\prime}$, there is no $t^{\prime} \in A-\left(Y^{\prime} \cup Z^{\prime}\right)$ such that $b \ldots t^{\prime} \ldots x \ldots=R^{\prime \prime \prime \prime}$ contradicting condition 2 of $W$. In consequence, $Y=Y^{\prime}$.

In the following, we prove that $Y \cup Z=Y^{\prime} \cup Z^{\prime}$. To the contrary let $Y \cup Z \neq Y^{\prime} \cup Z^{\prime}$. We will prove that $\left[\left(Y^{\prime} \cup Z^{\prime}\right) \times\left((Y \cup Z)-\left(Y^{\prime} \cup Z^{\prime}\right)\right)\right] \subseteq P$ which because of $\#\left(Y^{\prime} \cup Z^{\prime}\right) \geqslant 2$ clearly contradicts the assumptions on $P$. So let $x^{\prime} \in\left(Y^{\prime} \cup Z^{\prime}\right)$ and let $z \in\left((Y \cup Z)-\left(Y^{\prime} \cup Z^{\prime}\right)\right)$. Because of $Y=Y^{\prime}$ it follows that $z \in Z-Z^{\prime}$. Suppose to the contrary that $\left(x^{\prime}, z\right) \notin P$. Then there are $y^{\prime} \in Y$ and $R \in V$ with $y^{\prime} \ldots z \ldots x^{\prime} \ldots=R$. As $Y=Y^{\prime}$ both $x^{\prime}$ and $y^{\prime}$ are in $\left(Y^{\prime} \cup Z^{\prime}\right)$ Maskin $\left(\left(\left(Y^{\prime} \cup Z^{\prime}\right) \times Y^{\prime}\right) \cup I_{A}, W\right)$ implies the contradiction $z \in$ $\left(Y^{\prime} \cup Z^{\prime}\right)$. So, $\left(x^{\prime}, z\right) \in P$ and herewith $\left[\left(Y^{\prime} \cup Z^{\prime}\right) \times\left((Y \cup Z)-\left(Y^{\prime} \cup Z^{\prime}\right)\right)\right] \subseteq P$.

So, $Y=Y^{\prime}$ and $Z=Z^{\prime}$. Next we show that $P=P^{\prime}$ which then by the definition of $V$ and $W$ yields the desired result that $V=W$. First we show that $P \subseteq P^{\prime}$. To the contrary, suppose that $(a, b) \in P$ and $(a, b) \notin P^{\prime}$. Because $P \cup P^{\prime} \subseteq[(Y \cup Z) \times(A-Y)]$ and $((Y \cup Z) \times(A-(Y \cup Z))) \subseteq P \cap P^{\prime}$, it follows that $a \in Y \cup Z$ and $b \in Z$. Now because of the definition of $V$ there are $R \in V$ with bat... $=R$ for some $t \in(A-(Y \cup Z))$. Since $V \subseteq W$, this clearly yields a contradiction with condition 2 of $W$. So, $P \subseteq P^{\prime}$.

Next we prove $P^{\prime} \subseteq P$. Suppose $(a, b) \notin P$. We prove that $(a, b) \notin P^{\prime}$. Because $(a, b) \notin P$ it follows by the definition of $V$ that there are $R \in V$ such that both $y \ldots=R$ for some $y \in Y$ and ...b...a... As $R \in V \subseteq W$. This shows that $(a, b) \notin P^{\prime}$. 


\section{Conclusion}

By restricting the domain of only one agent, we showed that it is possible to escape the negative conclusions of the Gibbard-Satterthwaite and the Muller-Satterthwaite theorems. Obviously, the social choice functions we construct that are strategy-proof, Pareto-efficient and non-dictatorial/ Maskin monotonic, Pareto-efficient and non-dictatorial, indeed have a dictatorship flavor because of their hierarchical structures. ${ }^{11}$

A question of interest would be to study how these social functions evolve as we restrict the preferences of more than one agent. We leave this question open for future research.

We close the discussion with two examples. The first one shows that there are domains which admit non-dictatorial, Pareto-efficient and strongly positively associated choice rules but are not non-dictatorial, Pareto-efficient and strategy-proof choice rules.

Example 2 Let $B \varsubsetneqq A$, with $\# B \geqslant 3$, and let $t \in A-B$. Take $L^{i}=L(A)$ for $i \geqslant 2$ and $L^{1}=\left\{R \in L(A)\right.$ : if for some $b \in B R \in L_{b}(A)$, then there are $c \in B-\{b\}$ such that bct... $=R\}$. Define the choice rule $K$ from $L^{N}$ to $A$ for a profile $p$ as follows: $K(p)=a$ if $x \ldots=p(1), x=a$ and $x \notin B$ else xyt $\ldots=p(1)$ with $\{a, b\}=\{x, y\} \varsubsetneqq B$ and $\ldots a \ldots b \ldots=p(2)$.

Clearly $K$ is non-dictatorial, Pareto-efficient but not strategy-proof. Furthermore, $K$ is strongly positively associated. To show this consider $\{i\}-$ deviations $p$ and $q$ in $L^{N}$ such that $\left.p(i)\right|_{A-\{a\}}=\left.q(i)\right|_{A-\{a\}}$ and $l o w(a, p(i)) \subseteq$ low $(a, q(i))$. It is sufficient to show that $K(q) \in\{a, K(p)\}$. If $i>2$, then $K(p)=K(q)$. Let $i=2$. If $K(p) \notin B$, then $K(p)=K(q)$. Let $K(p) \in B$. Then $\{K(p), b\}=\{x, y\} \varsubsetneqq B$ for some $b, x, y$ in $A$ and $x y t \ldots=p(1)$ and $\ldots K(p) \ldots b \ldots=p(2)$. If $a \neq b$, then $\ldots K(p) \ldots b \ldots=q(1)$ and consequently $K(q)=K(p)$. If $b=a$, then obviously $K(q) \in\{K(p), a\}$. Let $i=1$. Let $K(p) \notin B$. If not $a \ldots=q(1)$, then $K(q)=K(p)$. If $a \ldots=q(1)$, then $a K(p) \ldots=q(1)$ and as $K(p) \notin B$ it follows that $a \notin B$. So, in that case $K(q)=a$. Let $K(p) \in B$. Then for some $x, y, b \in B$ we have $\{K(p), b\}=\{x, y\}, x y t \ldots=p(1)$ and $\ldots K(p) \ldots b \ldots=p(2)$. If $K(p)=a$, then $p(1)=q(1)$ or $q(1)=y x t \ldots$ and $K(p)=K(q)$. If $a \neq K(p)$ and not $a \ldots=q(1)$, then $K(p)=K(q)$. If $a \neq K(p)$ and $a \ldots=q(1)$, then $a K(p) b t \ldots=q(1)$. So, in that case $a \notin B$ and $K(q)=a$.

\footnotetext{
${ }^{11}$ Indeed, at many preference profiles, the agent with a restricted set of preferences gets his top alternative.
} 
Our last example shows that if we restrict the sets of preferences of exactly two agents, then neither the condition of inseparable pair nor the condition of inseparable set is a necessary condition for the maximality of a strategy-proof possibility domain.

Example 3 Let $A=\{x, y, z, t\}$ and $N=\{1,2\}$. Take $L^{1}=\{R \in L(A)$ : $R=x \ldots$ or $R=y \ldots x \ldots z \ldots$ or $R=t \ldots x \ldots z \ldots$ or $R=z \ldots\}$ and $L^{2}=\{R \in$ $L(A): R=x \ldots z \ldots t \ldots$ or $R=y \ldots t \ldots x \ldots$ or $R=z \ldots x$ or $R=t \ldots y \ldots x \ldots\}$. Now define the choice rule $K$ for an arbitrary profile $p \in L^{N}$ as follows $K(p)=a$ if $p(1)=a \ldots$ and $p(2)=b \ldots$ and $(a, b) \notin\{(y, x),(t, x)\}$ else take $K(p)=x$. So at $K$ agent 2 is decisive on the pairs $(x, y)$ and $(x, z)$, whereas agent 1 is decisive on the remaining pairs. So $K$ is not dictatorial. As $K(p)$ $\in\{\operatorname{best}(p(1))$, best $(p(2))\}$ we have that $K$ is Pareto-efficient. To see that $K$ is strategy-proof consider profiles $p$ with $p(2)=x \ldots$. Then the outcome for any $\{1\}$-deviation of $p$ is either $x$ or $z$ depending on whether agent 1 prefers $x$ to $z$ or $z$ to $x$ respectively. A similar reasoning holds for $\{2\}$-deviation of profile $p$ with $p(1)=y \ldots$ or $p(1)=t \ldots$. It is straight forward to see that for both agents $i$, the set $L^{i}$ does not have an inseparable pair or set. Therefore, we may conclude that these inseparabilities are not necessary conditions for maximal strategy-proof possibility domains in case the set of preferences of more than one agent is restricted. Note further that $K$ is tops-only but that the sets $L^{1}$ and $L^{2}$ are neither convex nor connected.

\section{References}

1. Barberà S., Gul F., Stacchetti E., 1993. "Generalized Median Voter Schemes and Committees." Journal of Economic Theory, 61, p. 262-289.

2. Barberà S., Masso J., Neme A., 1997. "Maximal Domains of Preferences preserving Strategy-Proofness for Generalized Median Voter Schemes." Social Choice and Welfare, 16, p.298-321.

3. Barberà S., Sonnenschein H, Zhou L, 1991. "Voting by Committees." Econometrica, 59, p. 595-609.

4. Ching S., Serizawa S., 1998. "A Maximal Domain for the Existence of Strategy-Proof Rules." Journal of Economic Theory, 78, 157-166.

5. Gibbard A., 1973, Manipulation of voting schemes: A general result, Econometrica, 41, p. 587-601.

6. Gibbard A., 1977, Manipulation of schemes that mix voting and chance, Econometrica, 45, p. 665-681. 
7. Gibbard A., 1978, Straightforwardness of game forms with lotteries as outcomes, Econometrica, 46, p. 595-614.

8. Kalai E. and Muller E., 1977. "Characterization of domains admitting nondictatorial social welfare

functions and nonmanipulable voting procedures." Journal of Economic Theory, 16, p.457 469.

9. Kalai E. and Ritz Z., 1980. "Characterization of the private alternatives domains admitting arrow

social welfare functions." Journal of Economic Theory, 22, p.23 36.

10. Masso J., Neme A., 2001. "A Maximal Domain of Preferences for the Division Problem." Games and Economic Behavior, 37, p.367-387.

11. Moulin H., 1980, On strategy-proof ness and single peakedness, Public Choice, 35, p.437-455.

12. Satterthwaite M.A., 1975, strategy-proof ness and Arrow's conditions: Existence and correspondence theorem for voting procedures and social welfare functions, Journal of Economic Theory, 10,p.187-217.

13. Serizawa S., 1995. "Power of voters and domain of preferences where voting by committees is strategy-proof." Journal of Economic Theory, 67, p.599 608 .

14. Storcken T., 1989."Possibility Theorems for Social Welfare Functions. " Tilburg University.

15. Zhou L., 1990, Impossibility of strategy-proof mechanisms in economies with pure public goods, Review of Economic Studies, 58, p.107-119. 